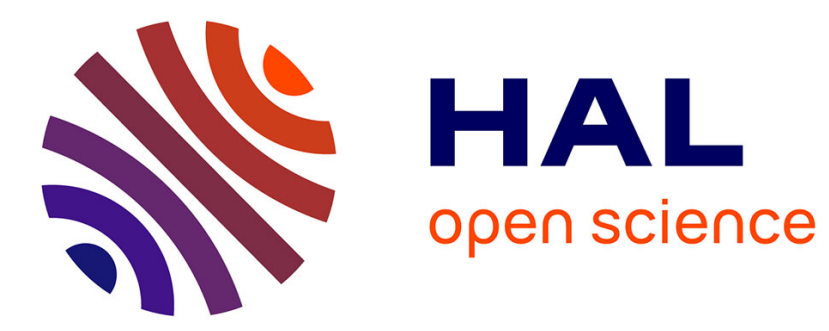

\title{
Une anomalie du fonctionnement de l'apex à hérédité mendélienne chez le pêcher (Prunus persica (L.) Batsch)
}

René Monet, Yves Bastard

\section{To cite this version:}

René Monet, Yves Bastard. Une anomalie du fonctionnement de l'apex à hérédité mendélienne chez le pêcher (Prunus persica (L.) Batsch). Agronomie, 1982, 2 (1), pp.103-106. hal-00884358

\section{HAL Id: hal-00884358 https://hal.science/hal-00884358}

Submitted on 1 Jan 1982

HAL is a multi-disciplinary open access archive for the deposit and dissemination of scientific research documents, whether they are published or not. The documents may come from teaching and research institutions in France or abroad, or from public or private research centers.
L'archive ouverte pluridisciplinaire $\mathbf{H A L}$, est destinée au dépôt et à la diffusion de documents scientifiques de niveau recherche, publiés ou non, émanant des établissements d'enseignement et de recherche français ou étrangers, des laboratoires publics ou privés. 


\section{Une anomalie du fonctionnement de l'apex à hérédité mendélienne chez le pêcher (Prunus persica (L.) Batsch)}

René MONET \& Yyes BASTARD

I.N.R.A., Station d'Arboriculture fruitière, Centre de Recherches de Bordeaux, F 33140 Pont de la Maye.

\section{RÉSUMÉ}

Pêcher,

Génétique mendélienne,

Anomalie morphogénéti-

que,

Fonctionnement de l'apex végétatif.
Un nouveau caractère, résultant d'une anomalie du fonctionnement des méristèmes apicaux, est décrit chez le pêcher. L'analyse génétique fait apparaître que ce phénotype appelé «triangle liégeux » est déterminé par un gène récessif et proviendrait d'un ancêtre commun : « Elberta ». Nous proposons les symboles génétiques : T pour l'allèle normal et $t$ pour l'allèle mutant.

\section{SUMMARY}

Peach,

Mendelian inheritance,

Morphogenetic anomaly, Shoot apex function.

\section{Simple mendelian inheritance of a shoot apex anomaly in peach}

A new character, resulting from anomalous function of the shoot apex, is described in peach. Genetic analysis showed that this phenotype " triangle liégeux = corky triangle " is dependent on a recessive factor and could be inherited from «Elberta». We suggest the symbols $T$ for the normal allele and $t$ for the mutant.

\section{INTRODUCTION.}

Le caractère que nous allons étudier a été déjà observé par SAUNIER (1968) sur certaines variétés de pêcher. Il se forme, chez les sujets atteints, à l'aisselle des feuilles, une zone nécrotique superficielle de forme triangulaire (fig. 1) avec, parfois, annulation des bourgeons axillaires du rameau. Sur un même rameau, plusieurs aisselles foliaires sont atteintes mais non toutes. On peut penser qu'il existe, au niveau de l'anneau initial, un fonctionnement anormal de certaines cellules génératrices. L'aspect de la nécrose suggère un triangle liégeux ce qui explique que l'on ait dénommé ainsi ce caractère.

Après avoir inventorié les variétés qui portent cette anomalie, SAUNIER tente d'en rechercher les causes. Il élimine ainsi l'action des températures, des viroses et du porte-greffe. Il examine aussi la possibilité de l'action d'un insecte, le thrips, et finalement ne peut prononcer de conclusion définitive. C'est en observant des arbres issus d'autofécondations contrôlées de certaines variétés que nous avons pu envisager l'origine génétique du caractère.

Figure 1

Aspect d'un "triangle liégeux» (flèche). La jeune pousse $(P)$ a été supprimée.

"Corky triangle" (arrow). The young shoot $(P)$ has been cut off.

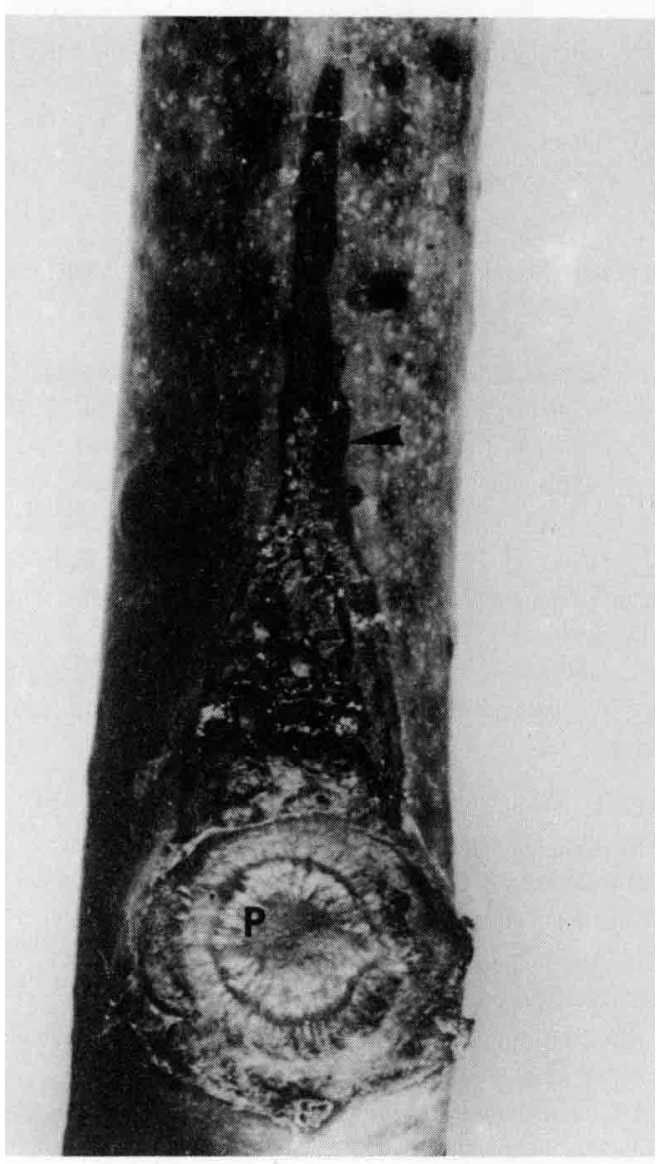


TABLEAU 1

Répartition des phénotypes dans les familles d'autofécondations Distribution of phenotypes in self-pollinated families

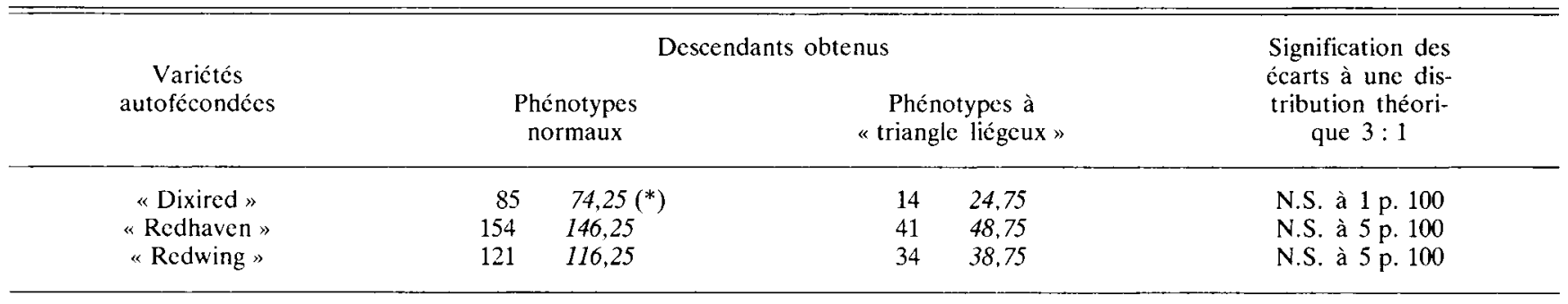

$\left.{ }^{*}\right)$ Les chiffres en italique sont les chiffres théoriques calculés pour une disjonction mendélienne $3: 1$.

II. OBSERVATION DU CARACTÈRE SUR TROIS FAMILLES OBTENUES PAR AUTOFÉCONDATION DE «DIXIRED », « REDHAVEN » ET « REDWING ».

L'observation attentive des arbres obtenus par l'autofécondation de ces 3 variétés nous a montré que certains d'entre eux manifestaient le triangle liégeux et que le caractère persistait d'unc année à l'autre sur les mêmes arbres sans se propager; l'anomalic n'était donc pas de nature épidémique. Par ailleurs, la fréquence des arbres atteints et des arbres normaux dans chaque famille était non significativement différente d'une disjonction mendélienne en monohybridisme, le caractère «triangle liégeux » ćtant récessif (tabl, 1). Ainsi, les géniteurs « Dixired», «Redhaven » et «Redwing » étaicnt hétérozygotes pour le caractère.

Pour avoir une nouvelle preuve de la nature héréditaire du caractère, nous avons autofécondé un arbre de la famille «Redhaven» portant le triangle liégeux (arbre $1161: 12$ ) et constaté que les 35 arbres obtenus ainsi portaient tous l'anomalie.

\section{ORIGINE GÉNÉALOGIQUE DE L'ANOMALIE}

Il est intéressant de signaler que les variétés répertoriées par SAUNIER comme présentant l'anomalic ont toutes, dans leur généalogie lorsque celle-ci est connue, comme ancêtre commun soit la variété «J. H. Hale», soit la variété «Elberta». Or, «J. H. Hale » est considérée comme issue d' "Elberta " (HEDRICK, 1917); on peut donc émettre l'hypothèse que ce dernier géniteur est la source à partir de laquelle le caractère a été dispersé.

Les variétés classées sensibles et très sensibles par SAUNIER (homozygotes vraisemblablement pour l'anomalie) sont :

« Richhaven» $=$ «Redhaven » $\times(« \mathrm{~J}$, H. Hale »

$x$ « Halehaven »)

«Summerrose » = «J. H. Hale » $x$ «Delicious »

«Madame Quès »: origine inconnue

« Nectared $2 »$ : hydride complexe dans lequel intervient

"Nectalate" dont un ancêtre est «Elberta ".

«Somervee» = «Halehaven» $(=« \mathrm{~J}$, H. Hale »

$\times$ «South Haven $») \times$ « Kalhaven»

«Early Elberta » (certains clones) : origine inconnue

«Elberta» (certains clones).
Dans notre hypothèse, «Richhaven» correspond de toute évidence au croisement entre 2 hétérozygotes ayant reçu chacun lanomalie de la source «Elberta" (voir plus bas la généalogic de "Redhaven"). En ce qui concerne «Summerrose », « Nectared 2 » et « Somervee », l'imprécision de la généalogie d'un de leurs géniteurs ne permet pas de dire si l'anomalie qu'ils portent provient d'une même origine («Elberta ») ou de deux.

Il existe enfin une ambiguité concernant «Elberta» puisque l'anomalie n'affecte, selon SAUNIER, que certains clones de cette variété.

Les variétés classées épisodiquement sensibles par le même auteur (hétérozygotes vraisemblablement pour l'anomalie) sont :

"Fairhaven " $=$ «J. H. Hale " $x$ «Southland»

«Redhaven» $=$ «Halehaven» $(=\langle\mathrm{J}$. H. Hale» $x$ "South Haven ") $\times$ « Kalhaven "

"Sunshine» = mutation de bourgeon de «Redhaven»

«Michelini » : origine inconnue

«Nectared $3 »$, « Nectared $6 »$ et « Nectared $10 »$ : hybrides complexes dans lesquels intervient toujours «Nectalate» dont un ancêtre est «Elberta ».

«Nectarine Cavalier»: origine inconnue.

A ces variétés, il faut ajouter «Dixired» et « Redwing» dont nous avons étudié la descendance et montré qu'elles étaient, avec «Redhaven», hétérozygotes pour le caractère; leur généalogie est la suivante:

«Dixired» = autofécondation de «Halehaven» $(=«$ J. H. Hale $x$ «South Haven $»)$

«Redwing $»=$ Babcock $X$ «Steengard July Elberta».

Le géniteur «Steengard July Elberta » est d'origine inconnue, il n'est pas nécessairement apparenté à «Elberta». De telle sorte que l'on ne peut pas dire si «Redwing» se rattache à la source "Elberta » de l'anomalie ou si elle a reçu ce gène d'une autre source qui pourrait être «Babcock».

Il faut noter que si des hétérozygotes, comme « Redhaven" par excmple, extériorisent épisodiquement le caractère, cela prouve que son expression est sensible aux conditions du milieu.

La présence, dans la généalogie, de la plupart des variétés étudiées par SAUNIER et par nous, d'un ancêtre commun, «J. H. Halc » ou «Elberta », n'est qu'une présomption sur l'origine du caractère; cette présomption ne peut devenir certitude que par l'établissement du génotype de chaque ascendant; notamment celui d' Elberta» (sur laquelle subsistent les incertitudes clonales) et de «J. H. Hale». 


\section{CONCLUSION}

L'ensemble des observations que nous venons de faire permettent de dire que le caractère observé est de nature héréditaire, qu'il est récessif et monogénique. Nous suggérons les symboles génétiques $\mathrm{T}$ pour désigner l'allèle normal et $\mathrm{t}$ pour désigner l'allèle mutant.
Ce caractère rend difficile la multiplication par greffage de l'arbre qui en est affecté, il est nécessaire que les sélectionneurs soient informés de sa nature et de son mode d'hérédité.

\section{RÉFÉRENCES BIBLIOGRAPHIQUES}

Hedrick U. P., 1917. The peaches of New York. J. B. Lyon Company printers Albany p. 234.
Saunier R., 1968. Quelques remarques concernant l'affection dite du «triangle liégeux» sur pêcher. Phytoma, n 200, 21-24. 